\title{
A method for producing random visual textures*
}

\section{JOHN B. PITTENGER and ANDREW P. KING Cornell University, Ithaca, New York 14850}

Textures composed of randomly shaped black and white areas may be produced from high-contrast photographs of a mixture of white and black-dyed rice. Moderate control of black-to-white ratio and many randomizations at a given ratio are possible. This method is cheaper than computer generation and better controlled than the more inexpensive methods.

Several methods are available for producing randomly textured surfaces for use in studies of visual perception. Flock (1964), for example, has described the application of powder or ink to electrostatically charged Plexiglas for use with the point-source shadow caster. In his studies of stereopsis, Julesz (1971) uses random dot patterns produced by computer on a cathode ray tube screen. The use of high-contrast photographs of a mixture of white and black-dyed rice has a number of advantages over these methods.

Viewed from a distance of several feet, such photographs are not recognizable as rice but, rather, appear to be composed of randomly shaped, two-dimensional patches of black and white. With this method, any desired black-to-white ratio can be produced, and it is easy to make any number of different randomizations at a given ratio. While the ink and powder methods are considerably cheaper, it is difficult to produce a specific black-to-white ratio in a single stimulus or to keep a constant ratio over a number of different stimuli. The computer-driven cathode ray tube gives excellent control of black-to-white ratios and has the further advantage of allowing variation of the ratio at will within different areas of a single stimulus. Programming and computer time are, however, quite expensive, and the equipment is not readily available to all workers. The rice photograph method is most useful when a number of fairly well-controlled textures are desired and computer generation is not feasible.

Black rice was prepared by dyeing uncooked rice (do not use "instant rice") in black cloth dye. Using one package of powdered dye (RIT No. 15 black, 1-1/8 oz) for each $2 \mathrm{lb}$ of rice, dissolve the dye in enough hot water to more than cover the rice. The rice should be allowed to soak for 1-2 h, and then drained and spread out on newspaper to dry. After the rice has dried, it will be covered with a fine black powder. This must be rinsed off so that the white rice is not discolored. To rinse the

*The development of this method was supported by a NIMH postdoctoral research fellowship to the first author. Requests for reprints should be sent to John B. Pittenger, Center for R esearch in Human Learning, 205 Elliott Hall, University of Minnesota, Minneapolis, Minnesota $\mathbf{5 5 4 5 5 .}$ rice, pour it into a bucket of warm water, stir, and immediately drain and dry. If the rinsing is done too slowly, the rice will crumble. The black rice may then be mixed with undyed white rice to give the desired black-to-white ratio.

The resulting mixture should be photographed with high-contrast film to give a print that is black and white without intermediate grays. Such grays produce brightness gradients corresponding to depth and give a print identifiable as rice. The texture in Fig. 1 was made by using Kodak High Contrast Copy Film (Improved) 5069.

To photograph the textures, spread the rice onto a flat surface large enough to give the desired density in the final print. Figure 1 used a $2 \times 3 \mathrm{ft}$ tray. Different randomizations may be made by stirring the rice thoroughly between exposures. A number of cautions should be kept in mind when using high-contrast film. Even illumination is important, since the film is sensitive to hot spots and shadows. We used four 400-W ECT photoflood lamps in 12-in. reflectors $4 \mathrm{ft}$ above the tray. As much light as possible should be used since high-contrast film is relatively slow and most $35-\mathrm{mm}$ cameras are not accurately calibrated for exposures longer than $1 \mathrm{sec}$. The rice should be patted flat to avoid hills and valleys which produce shadows. Since the film has little exposure latitude, it is best to make a test roll using 1/2-f-stop intervals around the expected correct exposure. Underexposure will produce gray around the edges of the rice, while overexposure will tend to fog the white areas. It is worth the extra effort to produce good negatives, since this will make it relatively easy to produce high-contrast prints.

Since high-contrast negatives are crucial to this

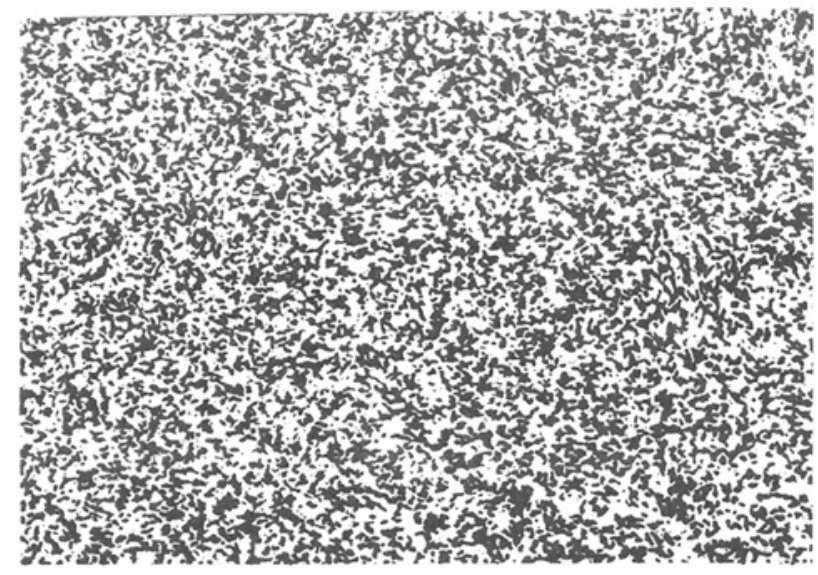

Fig. 1. Random texture composed of black and white grains of rice. 
method but difficult to make, details of the developing and printing methods are given below. The film was developed in Kodak D-19 developer for $6 \mathrm{~min}$, with agitation at 30-sec intervals, stopped in Kodak Indicator stop bath for $30 \mathrm{sec}$, fixed in Kodak Rapid Fixer for $1.2 \mathrm{~min}$, rinsed in water, washed in Kodak Hypo-Clearing Agent for $2 \mathrm{~min}$ with occasional agitation, and rinsed for $5 \mathrm{~min}$ in clear water. The temperature of all these baths was $68^{\circ} \mathrm{F}$. Finally, the film was dried in a clean, dust-free room.

The prints were made on $11 \times 14$ in. Kodak Polycontrast $\mathrm{N}$ paper. The film was printed almost full frame since this gives a virtually grainless print on this size paper. Polycontrast paper was chosen because it can be used with polycontrast filters to increase contrast with improperly exposed film. If, for example, the negative is lightly underexposed and has 5\% gray, exposing polycontrast paper through a No. 4 filter will eliminate the gray, leaving only black and white on the print. If the negatives are properly exposed, no filters are necessary.

The exposed paper can be developed in either Kodak Dektal or Kodak Ektaflo Type 1 (1:9) for 60-90 sec and stopped in either a Kodak Ektaflo or Kodak Indicator stop bath for 5.10 sec. Kodak Ektaflo Fixer should be used for $5.10 \mathrm{~min}$ in a single bath or for $3.5 \mathrm{~min}$ in a double bath and washed for $30-45 \mathrm{~min}$ in clear water. The prints should be dried in an electric dryer to produce a matte finish.

The choice between single- and double-weight paper depends on the degree of flexibility desired in the final print. Double-weight paper is recommended if the print is to be mounted, while single-weight is best if the print is to be bent into a curved surface. If the print is to be bent, a print flattening solution should be used in the developing process. For use in point-source shadow casters, the film can be printed onto clear acetate sheets.

\section{REFERENCES}

Flock, H. R. Irregular electrostatic surface-textures for use in the point-source shadow projector. American Journal of Psychology, 1964, 77, 318-320.

Julesz, B. Foundations of cyclopean perception. Chicago: University of Chicago Press, 1971.

(Received for publication November 8, 1972; accepted November 18, 1972.) 